\title{
Entertainment industry sector under the spotlight: How PR professionals and event organizers communicate during pandemic crisis
}

\author{
Andrei Galan \\ National University of Political Studies and Public Administration \\ Bucharest, Romania
}

\begin{abstract}
The COVID-19 pandemic has wounded sociality and limited the possibility to stay together: the essence of many forms of art. All the sectors whose activities are based on cultural values, or artistic, creative expressions, market or non-market oriented. It includes architecture, archives, libraries and museums, artistic crafts, audio-visual tangible and intangible cultural heritage, design, festivals, music, literature, performing arts, publishing, radio and visual arts. This paper aims to shed a light not only on the scarce literature on the field, but also on the particularities of how professionals communicate during pandemic crisis in the entertainment industry, especially at the moment when the whole world is being confronted by multiple crisis that have converged. In doing so, we explore how public relations practitioners and event organizers in Romania acknowledge the importance of being aware of the crisis, how they refer to crisis, the tools they use and the way they approach stakeholders. With the help of semi-structured interviews with public relations practitioners and event organizers in Romania, we aim to structure a frame of looking at the crisis communication in the entertainment industry during this pandemic crisis, with a particular focus on celebrities.
\end{abstract}

Keywords - Crisis, Entertainment Industry, Event Organizers, PR professionals, Sanitary crisis

SUGGESTED CITATION: Galan, A. (2021). Entertainment industry sector under the spotlight: How PR professionals and event organizers communicate during pandemic crisis. Proceedings of the International Crisis and Risk Communication Conference, Volume 4 (pp. 12-15). Orlando Fl: Nicholson School of Communication and Media. https://doi.org/10.30658/icrcc.2021.03

\section{INTRODUCTION}

When analysing the impact of the crisis we should acknowledge a variety of subsectors; it also includes all the activities of the cultural and creative products' value chain, from development to dissemination. The present work provides an updated assessment of the pandemic's economic impact on the sector, enlightening the intrinsic precarity of its workers whilst countries are confronting the second wave of infections. It continues with an updated panoramic overview of the measures taken at the international, national and local level to tackle the economic and social crisis.

The current environment is unprecedented and has had significant impacts on certain segments in the entertainment sector. Furthermore, outdoor consumption models - such as films, events and theme parks - are witnessing a dramatic fall with physical distancing norms in place. Overall ad-spend is determined by the performance of sectors such as fastmoving consumer goods, e-commerce, automotive, financial services, and real estate, all of which currently face their own challenges and could therefore take time to recover.

Getting ready for the crisis should be on the professional agenda of public relations practitioners, mainly when working with clients being on the spotlight. The overwhelming body of literature on both public relations [1] and crisis preparedness [2] seems to leave behind the specific challenges of managing public relations and crisis situation in entertainment industry [3], as they tend to ignore the interplay between professional and personal relationship of the practitioner and the client. Moreover, there is evidence that the practice of public relations for celebrities and public figures, both in terms of branding and crisis communication [4] is ahead of research. 
The practice of Public Relations for celebrities and public figures, both in terms of branding and crisis communication [4] is ahead of research as the literature on the field is still scarce [3]. For example, as it concerns the social media crisis, the academic research domain is still lacking certain theoretical models of crisis analysis and prevention. In the entertainment industry, crises are often perceived as disruptions of self presentation [5]. Most of the research papers in this domain emphasize the discursive strategies discussed by Benoit [6] (denial, evading responsibility, reducing offensiveness, coercive action, mortification) used by celebrities when dealing with reputational crisis. The research topics regarding celebrity PR crisis tend to be influenced by the nature of the previously mentioned theories, which emphasize the reputational impact of a crisis, leaving behind the research potential of the crisis preparedness and prevention stages.

The relevant literature identifies 5 main features that shape stakeholder's participation in a celebrity PR crisis: persistence, setting, replicability, scalability and searchability [5]. Still, no mention is made on the tools that PR specialists should use in order to analyse the impact of these features even from the pre-crisis stage.

\section{METHODS}

The method used for this research is represented by semi-structured interviews applied on ten PR Specialists and event organizers working for artists from the Romanian entertainment industry. The semi-structured interviews took place during $3^{\text {rd }}$ to $28^{\text {th }}$ of February 2021. All the participants on this study reported to have relevant studies regarding the entertainment industry as well as diplomas/certificates that justify the fact that they took part in trainings relevant to their professional domain. None of the respondents on this study were involved in a crisis at the time they participated on this study, but managed a reputational crisis involving their clients in the past months. The respondents had between 10-15 years of experience in the entertainment industry, studies and trainings specific to their markets and reported to have represented mainly artists from the music business. Between other PR activities (press relations, social media management, event management, branding) all the respondents reported to have been implied in the management process of at least one reputational crisis involving one of the celebrities whom they represented.

\section{RESULTS}

The crisis plan developed in the preparation stage usually includes real-time activities, messages and techniques based on intuition or on the experience gathered from managing similar situations in the past. In most of the cases, the usual crisis plan developed from the pre-crisis stage turns out to be a mental checklist that will be further updated during the crisis. Some of the most challenging triggers of a crisis in the entertainment industry refer to the situations emerging as a result of the different personality traits of the involved celebrity. Compared to brands (which have static identity elements whose risk can be prevented), celebrity crises express a higher level of unpredictability, as a result of the human factors involved in the process. You need to work on your relationship with the artist long before the crisis emerges. Even if they are public figures, at the end of the day they are humans as we are: they have problems and responsibilities which need to be anticipated and well-managed. (PR Specialist working for a singer, 15 years of experience).

The usual attributions of a crisis which triggers the entertainment industry mentioned by almost all the respondents include: responsibility, honesty, real-time reactions, pressure and unpredictability. Romanian PR specialists manually monitor by using free tools the online discussions mediums (press channels, forums, groups, chats) by searching the latest news using certain keywords related to the name of the celebrity they are representing. Public relations specialists barely interact with the negative comments, unless there is a hate wave that hits the celebrity. By monitoring the news, PR professionals mostly take reaction when it comes down to mass-media, by writing a press release in order to help their clients to establish the truth. PR professionals reported using Google Keyword Alerts as a main online visibility monitorization instrument.

When it comes to the point of approaching the stakeholders Emotion, Respect and Commitment: the 3 most important attributes that described the celebrity-artist relation, reporting to have a major importance when creating the crisis plan in the pre-crisis stage. The online mediums were reported to be more efficient than the offline ones (fan meetups/gatherings, concerts). During a crisis, the online community of the artists acts like a living organism that you can never totally control. This is why artists should make full use of the interaction and interactivity features when speaking to their publics, even before a potential crisis emerges (PR Specialist working for a singer, 10 years of experience)

On the other hand, the event organizers depicts a huge loss of business, employers livelihood at stake, revenue being affected globally and requirement of funds to attain stability in long run. The major statistics found in the research refer at the fact that $90 \%$ of the business being cancelled between March - October 2020, around 80\% companies will need to raise capital or debt from institutions or shareholders to survive. This facts show that the business in various sectors has seen a huge downfall due to this economic disruption. The major cause of this rising difficulties for the industry is either the increasing cancellation of events of 2020 or the postpone of these to 2021 worldwide. Furthermore, the daily wages workers, small and medium associates businesses are also facing brutal economic conditions leading for sudden support else there will be an increase in unemployment ratios. 


\section{DISSCUSSION}

Despite the ban on large gatherings, connection and social interaction are still an integral part of human life. Businesses have found novel and innovative ways to bring people together to share experiences, while still adhering to social distancing rules and guidelines. COVID-19 restrictions have forced us to rely on technology to connect with others and have been a catalyst for the rapid growth of companies that facilitate this. While services such as Zoom will likely not be quite as sought after when the pandemic is over, it looks like virtual events are here to stay, in one form or another. For example, "hybrid" events may become the norm, incorporating both live and virtual elements. This is a great opportunity for event planners, who will no longer be limited by the size of the venue and can expand their audience to include virtual attendees, as well as those who prefer to attend in person. Seven out of ten respondets of this reseach having facilites to upgrade certain events for thier customers online are using virtual medium in this pandemic. The reason is because of the losses they are inculcating and no certain get away from COVID-19 encouraged virtual events. Due to this, there is a certain increas in webinars, online discussions and live chats to keep the industry active.

The positive outcome for online live events are the increase in the sense of personalisation and ease of accessibility to the customers. In convetional events, there are lot of people and large area during performances which effects the experience of customers as they get disconnected or due to overcrowding. But, the events in this pandemic has overcome this hurdle through the opportunity of enjoying the event while staying at own premise. Another impact is the reformation of global village or getting close to each other by removing border barriers through this virtual growth. Though, people are waiting to pass this time rapidly but the events and festivals in this online manner has grown new opportunities for businesses around the globe and gave a sense of togetherness in this harsh time.

On the other hand, the impact of this sanitary crisis on advertising techniques are well seen as in this condition when people are confined to stay at their homes the usage of social media platforms have increased and methods have grown more consumer - centric, dynamic and engaging. This lead to organisation to promote more on these platforms and strengthen their services by claming to take proper care and sanitization measures. Event companies are also promoting their webinars, live events and chats through providing free masks, webinar certificates, engaging audiences through their COVID-19 measures and increasing their web traffics and participation. This is both positive and negative for the industry as the virtual events costs very less than conventional events which means low revenue also to the company due to the low charges. The usge of these forums affects the sectors and workers who are solely dependent on traditional manner of events leading to their unemployment.

\section{CONCLUSION}

The global sanitary crisis is the opportunity to acknowledge the importance of entertainment industry in nurturing social links, supporting mutual understanding and community engagement with a view to change behaviours and convince people that they are instrumental in solving problems. The pandemic has also stressed the economic importance of the sector notably festivals, live events, museums, cinemas or cultural retailing in making places and territories attractive to locals, tourists and investment. Life cultural experiences are greatly missed during the pandemic and digital streaming is a poor alternative to collective cultural gatherings. It shows that entertainment plays a crucial role in the well-being of communities and social cohesion. Culture is not simply entertainment or leisure time. It contributes to collective sensemaking. It helps humans define what matters as the globe is confronted with a mortal pandemic, a climate crisis and the threats of technological development to human values.

Undoubtedly the pandemic will generate great arts. This worldwide pandemic will inspire writing, bringing new literature, new cinematography and lyrics that will question our policies, technology, science and societal behaviours. In this section we attempt to describe challenges born from this crisis with a view to imagine policies required to enable entertainment industry to play a key role in an age that requires more global cooperation, the promotion of cultural diversity as well as solidarity. The crisis has been a formidable accelerator of existing trends notably the growth of digital networks, the market dominance of large media players, the emergence of new collective and individual behaviours. It also highlights the needs to call on artists and creative professionals to contribute to the recovery process and the future.

The future generation lives in the virtual world, a world that makes everything accessible and which does not require the traditional interactions. The new generation manipulates information at a faster pace and integrates such information with a minimum of cultural mediation. Access to culture is now the realm of search engines, digital servers and social media as much as cultural institutions. The scene means that big companies in the entertainment, culture and art sector will get bigger. More activity will flow into e-commerce and digital content platforms with large catalogue and marketing muscles (Disney, Amazon, Alphabet, Netflix, Tencent, and Apple). They will get bigger because they will have the financial resources and better access to capital support (from the financial sector) to buy their way out of the crisis. Cultural content will increasingly be bundled in special deals relying on users' data connecting retail, exhibition and digital distribution in well managed release patterns to maximize revenue streams. The risk is that cultural content, artists will primarily used as 'a promotional product" to sell other services and goods. For instance, you get free access to a subscription VOD service as long as you consume on an e-commerce site. Digital platforms and their ability to make 
the most of artificial intelligence will want to control the various exploitation steps and cut out the middleman. Audiences may not necessarily lose out as choices will increase but cultural mediation will be totally different. These might give more opportunity for talents to get funding with the risk of upsetting traditional business models and the value chain.

This research shows different impacts of COVID-19 on entertainment industry. The major effect of this pandemic is the severe loss in the industry due to huge cancellations and postponements. The pace of industry is very slow and surviving with the help of virtual events. The revenue and the workers are drastically impacted and require funds to survive and sustain at longer run. The innovative leaders of entertainment industry are working on different strategies like use of virtual technology to connect people and make them participate through organizing webinars, live chats $\&$ discussions, live artist performances and podcast. They are promoting these through different unique marketing strategies on social media platforms majorly. Therefore, the impact of this sanitary crisis on entertainment industry is severe and require support of governments and sponsors but they are still active and trying to survive through the means of virtual events.

\section{Author Biography}

Galan Andrei Alexandru, Ph.D Student, Associate Assistant Professor, National University of Political Studies and Public Administration, e-mail: andrei.galan@comunicare.ro

\section{REFERENCES}

[1] Sriramesh, K., \& Vercic Dejan. (2009). The global public relations handbook: theory, research, and practice. New York: Routledge.

[2] Cloudman, R., \& Hallahan, K. (2006). Crisis communications preparedness among US organizations: Activities and assessments by public relations practitioners. Public Relations Review, 32(4), 367-376 https://doi.org/10.1016/j.pubrev.2006.09.005

[3] Maiorescu, R. D. (2017). Personal public relations and celebrity scandals: A cross-cultural analysis of Twitter communication in the aftermath of Johnny Depp's accusations of domestic violence. Journal of Communication Management, 21(3), 254-266 https://doi.org/10.1108/JCOM-02-2017-0006

[4] Davis, L., J., (2013) Crisis Tales: Five Rules for Coping with Crises in Business, Politics, and Life. New

[5] Colapinto, C., \& Benecchi, E. (2014). The presentation of celebrity personas in everyday twittering: Managing online reputations throughout a communication crisis. Media, Culture, and Society, 36(2), 219-233 https://doi.org/10.1177/0163443714526550

[6] Benoit, W. (1994). Accounts, Excuses, and Apologies: A theory of image restoration strategies. State University of New York Press. 